\title{
IL-36 receptor deletion attenuates lung injury and decreases mortality in murine influenza pneumonia
}

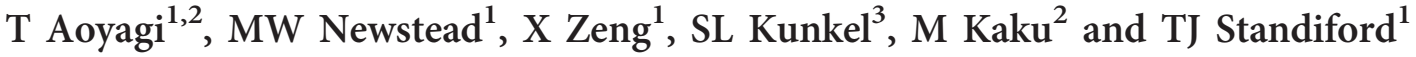

Influenza virus causes a respiratory disease in humans that can progress to lung injury with fatal outcome. The interleukin (IL)-36 cytokines are newly described IL-1 family cytokines that promote inflammatory responses via binding to the IL-36 receptor (IL-36R). The mechanism of expression and the role of IL-36 cytokines are poorly understood. Here, we investigated the role of IL-36 cytokines in modulating the innate inflammatory response during influenza virus-induced pneumonia in mice. The intranasal administration of influenza virus upregulated IL-36 $\alpha$ mRNA and protein production in the lungs. In vitro, influenza virus-mediated IL-36 $\alpha$ but not IL-36 $\gamma$ is induced and secreted from alveolar epithelial cells (AECs) through both a caspase-1 and caspase-3/7 dependent pathway. IL-36 $\alpha$ was detected in microparticles shed from AECs and promoted the production of pro-inflammatory cytokines and chemokines in respiratory cells. IL-36R-deficient mice were protected from influenza virus-induced lung injury and mortality. Decreased mortality was associated with significantly reduced early accumulation of neutrophils and monocytes/macrophages, activation of lymphocytes, production of pro-inflammatory cytokines and chemokines, and permeability of the alveolar-epithelial barrier in despite impaired viral clearance. Taken together, these data indicate that IL-36 ligands exacerbate lung injury during influenza virus infection.

\section{INTRODUCTION}

Influenza virus-induced pneumonia and resultant acute respiratory distress syndrome are associated with high mortality in critical care patients. ${ }^{1}$ High viral load, excessive pro-inflammatory cytokines and chemokine expression and robust inflammatory cells influx into the lungs are associated with disease severity in influenza virus infection. ${ }^{2-4}$ Imbalance between viral pathogenicity and host immune response is believed to contribute to influenza virus-induced respiratory failure.

Interleukin (IL)-36 cytokines, including three agonist proteins IL-36 $\alpha$, IL-36 $\beta$, and IL-36 $\gamma$, along with the antagonist protein IL-36Ra, are novel members of IL-1 family of cytokines. IL-36 agonists bind the same receptor complex, consisting of the IL-36 receptor (IL-36R) and IL-1 receptor accessory protein (IL-1RAcP), a protein that is shared with the IL-1 receptor and the IL-33 receptor. Binding to this complex stimulates inflammatory signals through nuclear factor- $\kappa \mathrm{B}$ and mitogen-activated protein kinase. ${ }^{5}$ The IL-36 agonists similarly induce pro-inflammatory cytokines and chemokines and contribute to neutrophils accumulation, dendritic cell activation, and polarization of $\mathrm{T}$ helper 1 and IL-17-producing T cells. ${ }^{5-7}$ IL-36 cytokines are expressed by keratinocytes and other epithelial cells, as well as macrophages and monocytes. Conversely, IL-36R is widely expressed throughout the body. ${ }^{5}$ It was well characterized that IL-36 cytokines are involved in the pathogenesis of human psoriasis ${ }^{8,9}$ and animal models of skin inflammation. ${ }^{10,11}$ However, little is known about the role of IL36 cytokines in the lung. Transcript products of IL-36 $\gamma$ have been shown to be upregulated in bronchial epithelial cells by stimulation of pro-inflammatory cytokines, bacteria, and viralassociated molecular patterns. ${ }^{12,13}$ Intratracheal administration of IL-36 $\alpha$ or IL-36 $\gamma$ in mice induce a rapid influx of neutrophils into the lungs and the expression of proinflammatory cytokines and chemokines. ${ }^{14,15}$ These previous findings suggest that IL-36 cytokines may play an important

${ }^{1}$ Division of Pulmonary and Critical Care Medicine, Department of Internal Medicine, University of Michigan, Ann Arbor, Michigan, USA. ${ }^{2}$ Department of Infection Control and Laboratory Diagnostics, Internal Medicine, Tohoku University Graduate School of Medicine, Sendai, Japan and ${ }^{3}$ Department of Pathology, University of Michigan, Ann Arbor, Michigan, USA. Correspondence: T Aoyagi (tetsujiaoyagi@med.tohoku.ac.jp) 
role in host defense against respiratory pathogens and contribute to leukocyte recruitment during infection. To date, no studies have investigated IL-36 cytokines in the host defense against influenza virus or as mediators of virus-induced lung injury.

Secretion mechanism of IL-36 cytokines from stimulated cells is not well understood. IL-36 cytokines, like other IL-1 family cytokines, lack a signal peptide and do not utilize a classical endoplasmic reticulum-to-Golgi secretion pathway, requiring alternative mechanisms for their release. Extracellular adenosine triphosphate (ATP) has been shown to be necessary for the secretion of intracellularly produced IL-36 cytokines. ${ }^{8,16,17}$ Stimulation of $\mathrm{P} 2 \mathrm{X} 7$ receptor by extracellular ATP induced NOD-like receptor family, pyrin containing 3 (NLRP3) inflammasome activation, and caspase-1-dependent processing and release of IL-1 $\beta$ and IL- $18 .{ }^{18}$ Moreover, P2X7 signaling induces the release of extracellular microvesicles or microparticles (MPs), and secreted IL-1 $\beta$ and IL-18 are packaged within MPs. ${ }^{19,20}$ MPs are membrane-bound vesicles ranging in size from 0.1 to $1 \mu \mathrm{m}$ released by a variety of cells. MPs contain proteins, bioactive lipids, nucleic acid, and organelles, and are recognized to be a biomarker and effector of cell-to-cell signaling in inflammatory diseases. ${ }^{21}$ We have recently shown that IL-36 $\gamma$ protein shed from macrophages stimulated with bacteria and ATP was detected within MPs. ${ }^{22}$ However, the role and secretion mechanism of IL-36 cytokines in the pathogenesis of lung inflammation is unknown.

In the current study, we hypothesized that IL-36 cytokines and their receptor, IL-36R, can contribute to the pathogenesis of influenza virus-induced lung injury. Influenza virus infection in mice resulted in the selective upregulation of IL-36 $\alpha$, but not IL-36 $\beta$ and $\gamma$, in the lungs. IL-36 $\alpha$ was secreted by alveolar epithelial cells (AECs) in MPs, exosomes (EXs), and directly into the extracellular milieu in response to influenza virus, and AEC-derived MPs directly stimulate cytokine and chemokine production in pulmonary macrophages (PMs) in vitro in an IL36R-dependent fashion. As compared with wild-type (WT) controls, IL-36 $\mathrm{R}^{-1-}$ mice display decreased early accumulation and activation of inflammatory cells, production of proinflammatory cytokines and chemokines, and reduced alveolar-epithelial barrier leak, which is associated with reduced mortality in response to influenza virus challenge. Taken together, these data demonstrate for the first time an important role of IL-36R ligands in the pathogenesis of influenza virus-induced lung injury.

\section{RESULTS}

IL-36 $\alpha$ is selectively induced in the lungs during influenza virus infection

We first examined the transcript products of IL-36 cytokines in the lungs of $\mathrm{WT}$ C57B/6 mice infected with influenza virus (Influenza A/Puerto Rico/8/34 virus). Both IL-36 $\alpha$ and IL-36 $\gamma$ mRNA levels were significantly elevated in lung at day 2 after influenza administration, and this increase in IL-36 $\alpha$ mRNA, but not IL-36 $\gamma$ mRNA, persisted through day 6 (Figure 1a). Conversely, IL-36 $\beta$ mRNA was not detected in influenza virus- infected lungs (data not shown). The protein levels of IL-36 $\alpha$ in

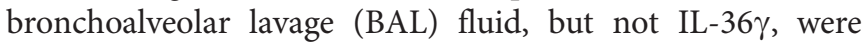
significantly higher at day 2 compared with untreated mice (Figure 1b).

We have recently shown that IL-36 $\gamma$ protein released from primary PMs stimulated with Streptococcus pneumoniae was primarily packaged within MPs and EXs. We therefore examined whether IL-36 $\alpha$ in BAL fluid post influenza virus challenge was present in MPs and EXs. To assess this, BAL was performed at day 3 after flu administration, then MPs and/or EXs in BAL fluid were isolated by three-step centrifugation. ${ }^{22}$ IL-36 $\alpha$ was detected by enzyme-linked immunosorbent assay within the MPs, EXs, and post-spin fluid fraction, and was significantly higher in the MP and post-spin fractions in influenza virus-infected mice as compared with untreated mice (Figure 1c).

\section{IL-36 $\alpha$ is released from influenza virus stimulated AECs through caspase-1- and caspase-3/7-dependent pathways} Respiratory epithelial cells and macrophages are important innate immune cells during influenza virus infection. To determine the primary cellular source of IL-36 $\alpha$ during influenza, primary AECs and PMs isolated from WT mice were incubated with various doses of influenza virus. Lipopolysaccharide (LPS) stimulation was used as a control immune activator. Treatment with LPS induced IL-36 $\alpha$ mRNA in both AECs and PMs. Though the expression level of IL-36 $\alpha$ mRNA was significantly elevated in influenza virus-treated AECs in a dose dependent manner, IL-36 $\alpha$ mRNA was not induced in PMs by influenza virus (Figure 2a). Heat killed (fusion inhibiting) influenza virus failed to induce IL-36 $\alpha$ mRNA in AECs (data not shown). Incubation with ultravioletinactivated influenza virus (replication inhibiting)-induced IL$36 \alpha$ mRNA in AECs; its level was significantly lower as compared with live virus (see Supplementary Figure S1A online).

Two distinct toll-like receptors (TLRs)-3 and TLR-7 recognize the double stranded and single stranded viral RNA of influenza virus, respectively, within endosomes and play key roles in the innate immune responses against virus. ${ }^{23}$ To examine whether these pathogen recognition receptors contribute to induction and secretion of IL-36 $\alpha$, we first compared mRNA expression of IL-36 $\alpha$ in AECs treated with the TLR-3 agonist poly (I:C); the TLR-7 agonist R843; the combination of poly (I:C) plus R843; influenza virus; or LPS. Unlike a previous study, ${ }^{17}$ treatment with poly (I:C) alone did not induce IL-36 $\alpha$ mRNA. Conversely, treatment with R843 alone enhanced expression of IL-36 $\alpha$ mRNA, and its level tended to be enhanced by co-stimulation with poly (I:C) treatment (Figure $2 \mathbf{b}$ ). To evaluate secretion of IL-36 $\alpha$ protein, IL-36 $\alpha$ was measured in culture medium (CM) by enzymelinked immunosorbent assay. The production of IL-36 $\alpha$ was significantly elevated in AECs treated with live influenza virus or with the combination of poly (I:C) plus R843, but not poly (I:C) or R843 alone (Figure 2c). These data suggest that both TLR-3 and TLR-7 signaling were required for secretion of 

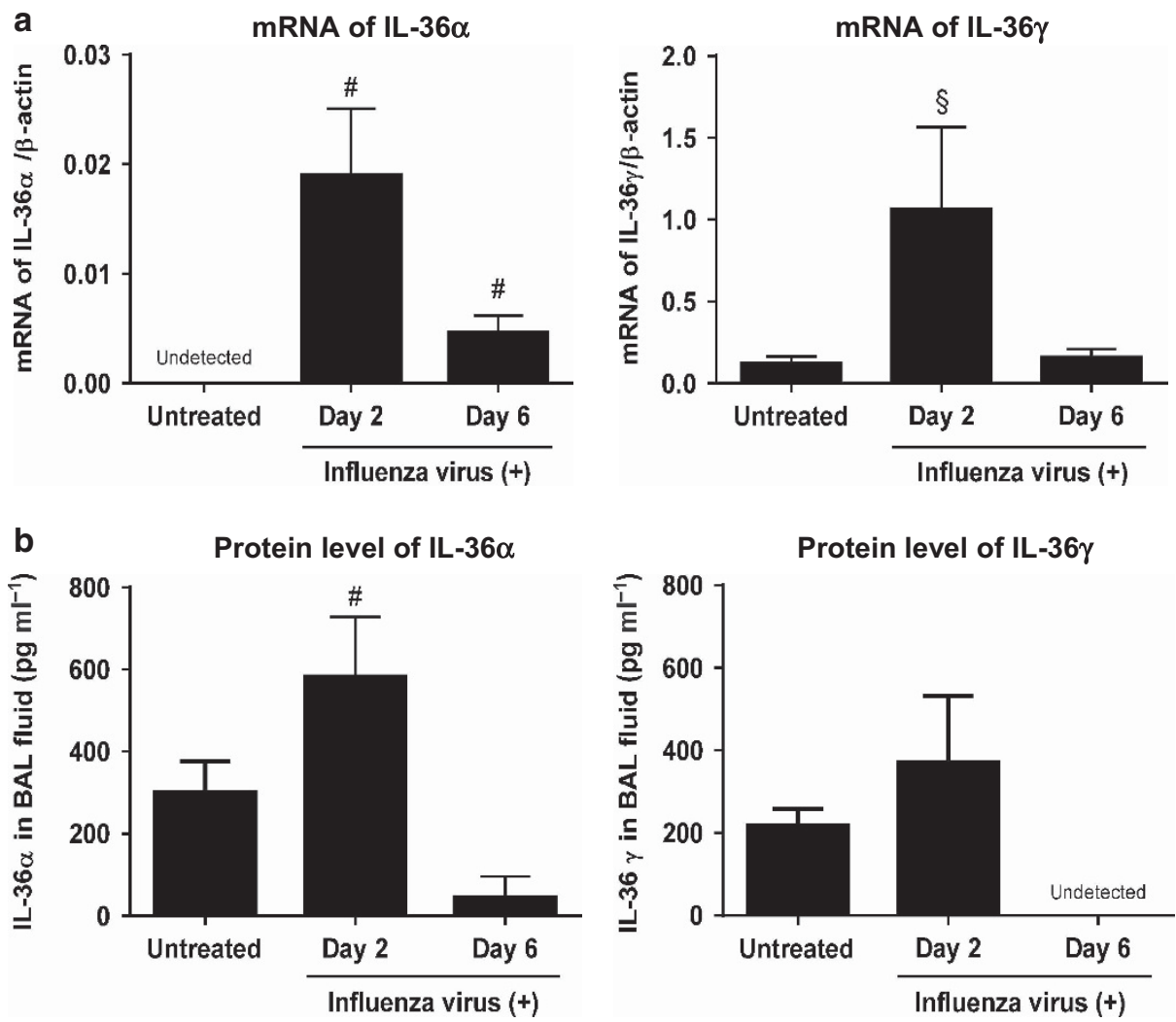

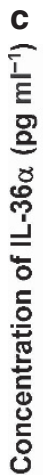
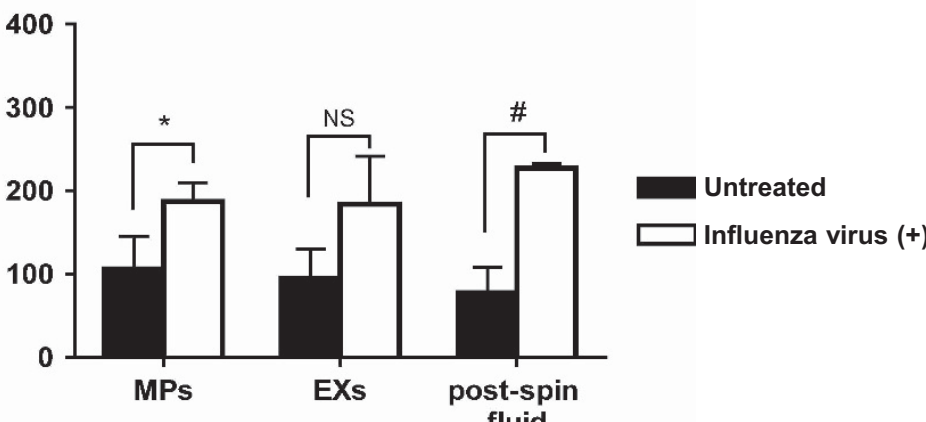

Figure 1 Expression of IL-36 cytokines in lung of WT mice infected with influenza virus. (a) Transcript products of IL-36 $\alpha$ (left panel) and IL-36 $\gamma$ (right panel) in the lungs of untreated, day 2, and day 6 after influenza virus infection. mRNA was analyzed by real-time PCR. (b) Protein expression of IL-36 $\alpha$ (left panel) and IL-36 $\gamma$ (right panel) in BAL was quantified by ELISA. (c) MPs and EXs in BAL fluid were isolated by three-step centrifugation. Production of IL-36 $\alpha$ in MPs, EXs and post-spin fluid in which MPs and EXs fractions were removed were measured by ELISA. Data are shown as means \pm s.d. of five mice per group. ${ }^{*} P<0.05,{ }^{\#} P<0.01,{ }^{\S} P<0.001$, significant compared with untreated mice or as indicated. BAL, bronchoalveolar lavage; ELISA, enzymelinked immunosorbent assay; IL-36, interleukin-36; WT, wild type.

IL-36 $\alpha$ by AECs. Moreover, we did not observe secretion of IL-36 $\alpha$ by AECs incubated with ultraviolet -inactivated influenza virus (see Supplementary Figure S1B). These data indicate that viral replication is required for maximal induction and secretion of IL-36 $\alpha$ from virus-infected AECs.

We next investigated the influence of influenza-induced caspase- 1 and caspase-3/7 activation on induction and secretion of IL-36 $\alpha$. Influenza virus has previously been shown to induce the expression of IL-1 like family members such as IL$1 \beta$ and IL-18 in respiratory cells by a caspase-1-dependent manner. ${ }^{18}$ Moreover, the secretion of IL-36 cytokines from stimulated cells has been shown to require extracellular ATP treatment (which activates caspase-1) ) $^{8,16,17}$ or caspase-3/7 activity. ${ }^{17}$ We confirmed that live influenza virus activated caspase- 1 and caspase-3/7 in AECs (see Supplementary Figure 2C, D). While treatment with caspase-3/7 inhibitor did not alter IL-36 $\alpha$ mRNA levels (Figure 2d), the secretion of IL-36 $\alpha$ in CM was significantly reduced by caspase-3/7 inhibitor (Figure 2e). Alternatively, caspase-1 inhibition suppressed both mRNA and extracellular protein expression of IL-36 $\alpha$ (Figures $2 \mathbf{d}$ and $\mathbf{e}$ ). These data indicated that live influenza virus-induced caspase-1 activation contributed to 

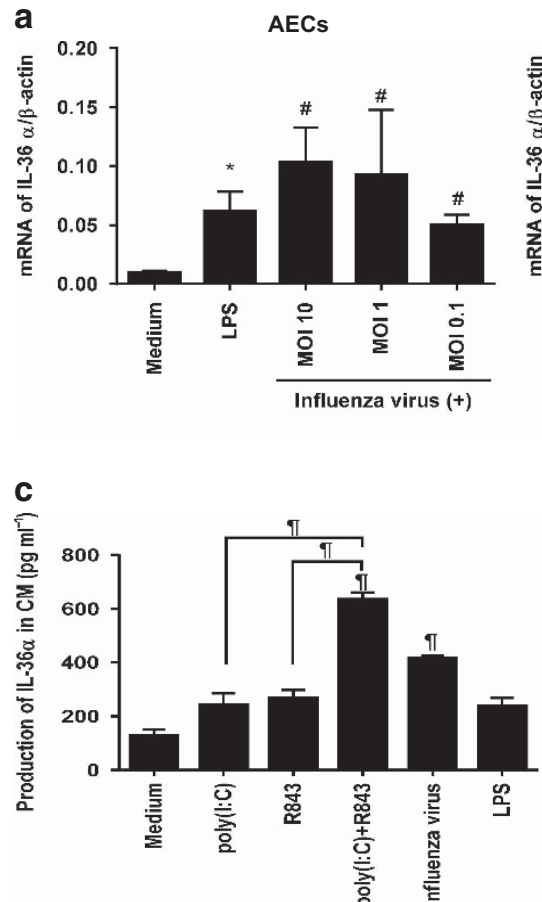

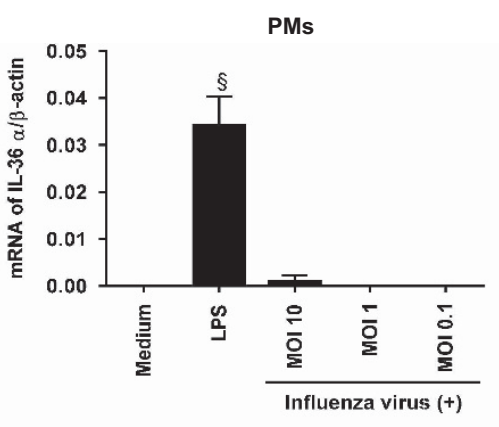

d

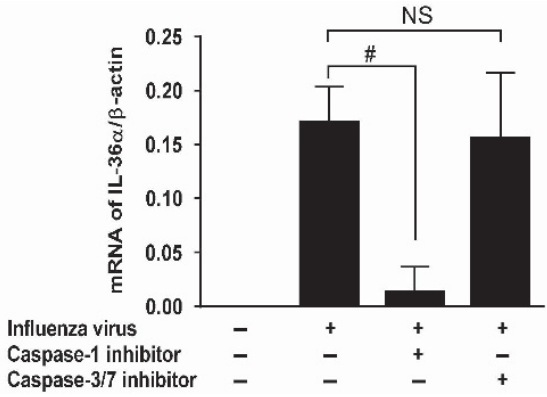

b

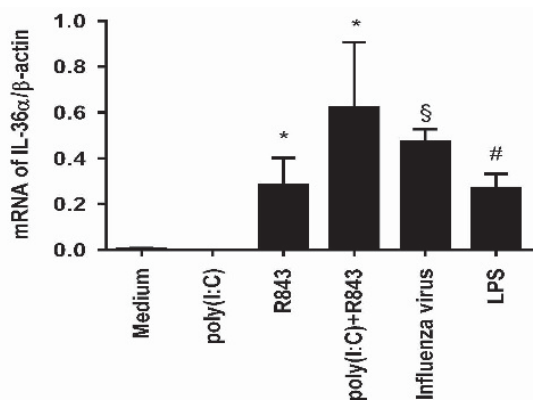

e

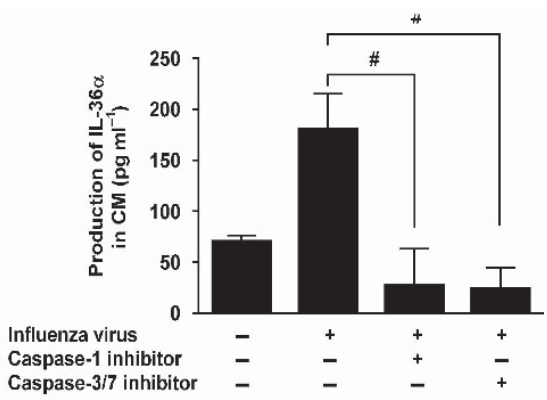

Figure 2 Mechanisms of IL-36 $\alpha$ protein synthesis and secretion from AEC in response to influenza virus, TLR-3, and TLR-7 agonists. (a) Primary AECs (right panel) and PMs (left panel) isolated from WT mice were treated with LPS (1 mg ml${ }^{-1}$ ) or different doses of influenza virus (MOI 10-0.1). After 24 $\mathrm{h}$ incubation, expression of IL-36 $\alpha \mathrm{mRNA}^{-1}$ was analyzed by real-time PCR. (b,c) AECs were treated with poly (I:C) $\left(25 \mu \mathrm{g} \mathrm{ml}^{-1}\right), \mathrm{R}^{-1} 43\left(25 \mu \mathrm{g} \mathrm{ml}^{-1}\right)$, both poly


real-time PCR. (c) The protein levels of IL-36 $\alpha$ in CM were measured by ELISA. (d,e) AECs were pre-treated with Caspase-1 (20 $\mu \mathrm{m})$ or Caspase-3/7 $(100 \mu \mathrm{M})$ inhibitor $1 \mathrm{~h}$ before influenza virus stimulation. After $24 \mathrm{~h}$ incubation, IL-36 $\alpha \mathrm{mRNA}$ (d) and protein production in CM (e) were analyzed. Data (means \pm s.d.) are representative of two independent experiments. ${ }^{*} P<0.05,{ }^{\sharp} P<0.01,{ }^{\circledR} P<0.001,{ }^{\natural} P<0.0001$, NS, not significant, compared with medium only or as indicated. AEC, alveolar epithelial cell; CM, culture medium; ELISA, enzyme-linked immunosorbent assay; IL-36, interleukin-36; LPS, lipopolysaccharide; MOI, multiplicity of infection; PM, pulmonary macrophage; TLR, toll-like receptor; WT, wild type.

induction of IL-36 $\alpha$, whereas caspase-3/7 was required for the secretion of IL-36 $\alpha$ from AECs. Interestingly, we did not observe apoptosis of AECs at the $24 \mathrm{~h}$ post infection (as determined by Annexin $\mathrm{V}+$ staining), a time point in which IL-36 $\alpha$ was detected in conditioned media (data not shown), suggesting that caspase- 1 and caspase- $3 / 7$ activity, but not cell death, is required for the induction and secretion of IL-36 $\alpha$ in influenza virus-infected AECs.

\section{Influenza virus induced IL-36 $\alpha$ from AECs is contained within MPs}

As shown previously, a percentage of IL-36 $\alpha$ in BAL was secreted within MPs during influenza virus infection (Figure 1c), and the secretion of IL-36 $\alpha$ from AECs was dependent upon influenza virus-induced activation of caspase3/7 (Figure 2e). We next examined whether IL-36 $\alpha$ secreted from influenza virus-treated AECs was packaged within extracellular vesicles. AECs were treated with or without influenza virus for $48 \mathrm{~h}$, then MPs and EXs isolated from CM by three-step centrifugation. Although there was no difference of number of MPs released by influenza virus-treated AECs as compared with vehicle-treated cells, the size of MPs shed from influenza virus-treated AECs were larger than that from untreated AECs (Figure 3a). On the other hand, no difference was observed in the number and size of EXs between the two groups. As shown Figure $3 \mathbf{b}$, the quantity of IL- $36 \alpha$ protein contained within influenza virus-induced MPs was $>2$-fold higher than that of MPs recovered from uninfected cells, whereas no difference in IL-36 $\alpha$ levels was observed in EXs and post spin fluid (Figure 3c). To address the size difference in MPs shed from influenza virus-infected cells as compared with untreated control, we examined whether or not influenza virus was contained within MPs. MPs were isolated from CM with or without influenza virus-treated AECs by ultracentrifugation and then washed twice to remove virus binding to the surface of MPs. Immunoblotting showed the presence of both IL-36 $\alpha$ and influenza virus M2 protein within MPs from influenza-infected AECs (Figure 3d), and virus contained with these MPs could be grown in MDCK cells (Figure 3e).

\section{IL-36R ${ }^{-1-}$ mice were protected from influenza virus- induced lung injury and mortality}

To examine whether IL-36R-mediated signaling is involved in the host response against influenza infection, WT IL-36R $\mathrm{R}^{-1-}$ were inoculated intranasally with $2 \mathrm{LD}_{50}$ dose of influenza virus, survival and body weight change assessed out to 14 days. Because IL-36 cytokine have previously been shown to enhance the expression of other IL-36 family members, ${ }^{11}$ and we observed upregulation of IL-36 $\gamma$ mRNA in lungs of influenza virus infected mice at day 2 , we included IL- $36 \gamma^{-1-}$ as an 
a
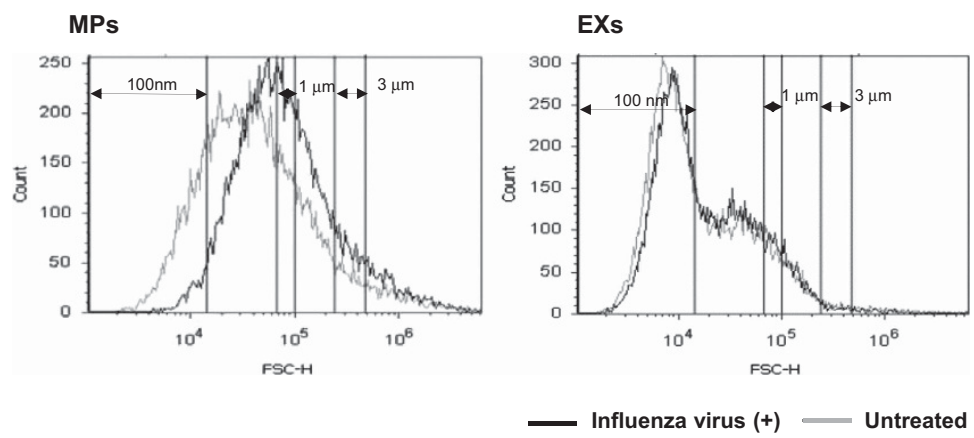

b

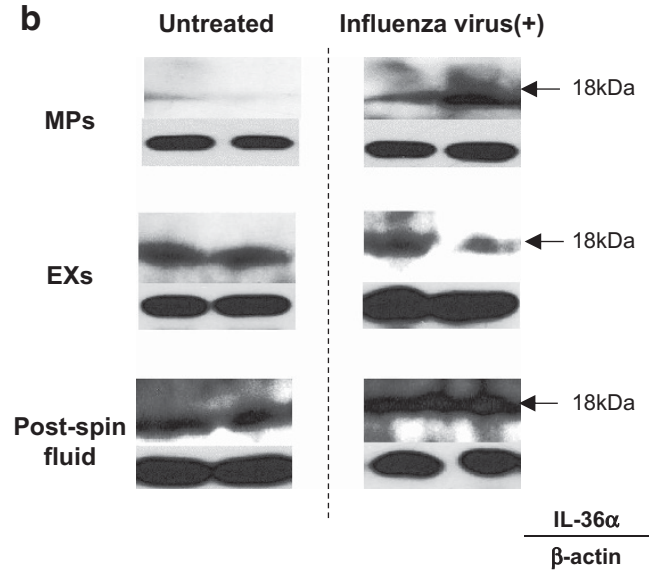

d

IL-36 $\alpha$

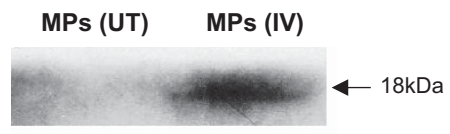

Influenza virus M2 protein

$\beta$-actin

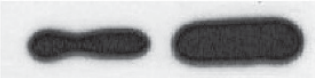

e

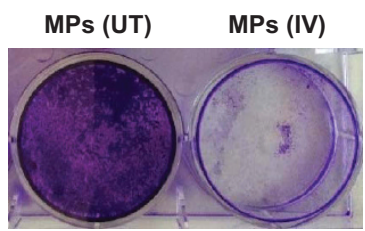

Figure 3 IL-36 $\alpha$ released from influenza virus stimulated AECs contained within MPs. AECs were treated with or without influenza virus for $48 \mathrm{~h}$ incubation, and then MPs and EXs were isolated from CM by three-step differential centrifugation. (a) MPs and EXs were identified as Annexin V-positive cells. The size of MPs (left panel) and EXs (right panel) was assessed by flow cytometry for size relative to submicron calibration beads. (b) The protein expression of IL-36 $\alpha$ in MPs, EXs, and post-spin fluid was detected by western blotting. Arrows showed the expression of IL-36 $\alpha$ at $18 \mathrm{kDa}$ (upper panel). Lower panel showed $\beta$-actin at $42 \mathrm{kDa}$. (c) The production of IL-36 $\alpha$ in MPs, EXs, and post-spin fluid were measured by ELISA. Data are shown as means \pm s.d.. ${ }^{*} P<0.05$, NS, not significant as indicated. (d, e) MPs isolated from CM were washed twice to remove surface-bound virus. The protein expression of IL-36 $\alpha$ and influenza virus M2 antigen in MPs were detected by western blotting. Arrow shows the expression of IL-36 $\alpha$ at $18 \mathrm{kDa}$ (upper panel), influenza virus M2 antigen at $15 \mathrm{kDa}$ (middle panel), and $\beta$-actin at $42 \mathrm{kDa}$ (lower panel) (d). MPs were cultured with MDCK cells (e). AEC, alveolar epithelial cell; CM, culture medium; ELISA, enzyme-linked immunosorbent assay; EX, exosomes; IL-36 $\alpha$, interleukin-36 $\alpha$; MP, microparticle; MPs (UT), MPs from untreated AECs; MP (IV), MPs from influenza virus-treated AECs.

additional control group. Body weight was not significantly different among the three groups (Figure 4a). However, all of WT and IL-36 $\gamma^{-1-}$ mice were dead within 11 days post infection, whereas $50 \%$ of IL-36R $\mathrm{R}^{-1-}$ mice survived beyond day 14 post infection (Figure $4 \mathbf{b}$ ). We next examined viral clearance after influenza virus challenge by quantitating lung viral titers in WT and IL-36R $\mathrm{R}^{-1-}$ mice on days 2 and 6 after infectious challenge. There was no difference in viral titers observed between WT and IL-36R ${ }^{-1-}$ mice at day 2 (Figure 4c, left panel). However, by day 6 there was a 10 fold higher viral burden in IL-36 $\mathrm{R}^{-1}$ mice as compared with infected WT mice (Figure 4c, right panel).

Histological examination of lungs on day 2 post influenza virus administration revealed accumulation of neutrophils in a peribronchial distribution in WT mice, and no difference in inflammatory cells influx into alveolar space between WT and IL-36R $\mathrm{R}^{-1-}$ mice at that time point. At day 7 in WT mice, there was massive infiltration of neutrophils not only into the intraalveolar septa but into the alveolus, as well as alveolar hemorrhage, alveolar septa edema, and proteinaceous debris in the alveolar space. These inflammatory changes were strikingly attenuated in IL-36 $\mathrm{R}^{-1-}$ mice. We quantified the histological changes of tissue injury using a standardized lung injury scoring system. ${ }^{24}$ The lung injury score was significantly lower in IL-36R $\mathrm{R}^{-1-}$ mice as compared with infected WT mice (Figure 4e). We next assessed the permeability of the alveolar-capillary membrane by measurement of albumin concentration in BAL fluid. At 

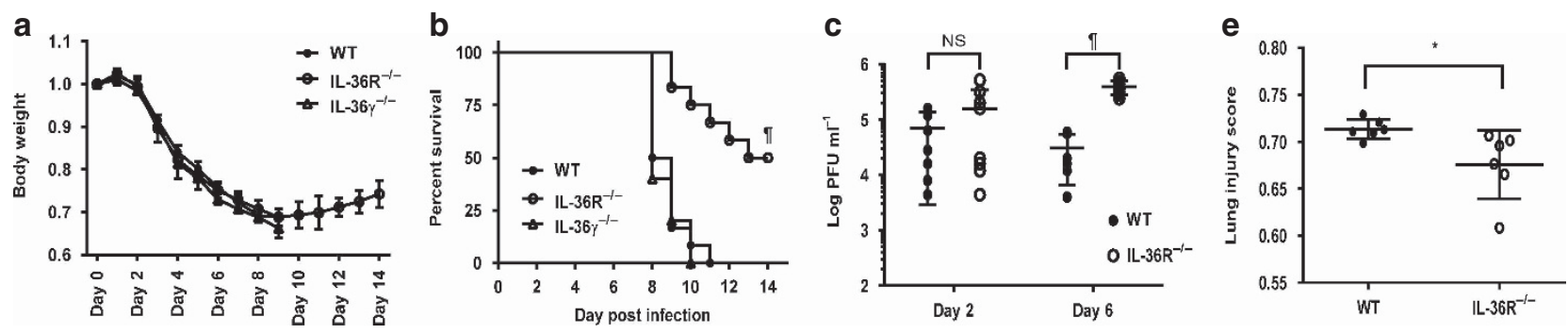

d
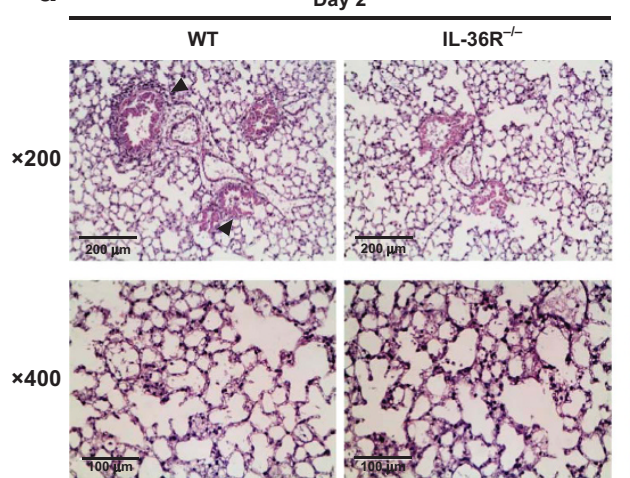


f

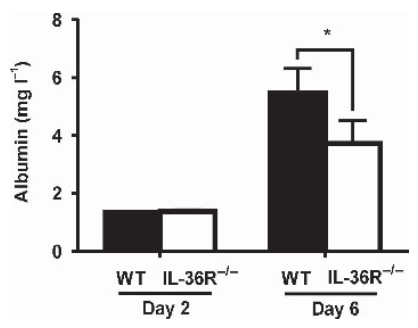

Figure 4 Effect of IL-36R gene deletion on mortality, viral clearance, and lung injury in influenza virus infection in vivo. Weight loss (a) and survival rate (b) were assessed daily following influenza virus infection. Each group consisted of 6-12 mice. (c) Virus titers was assessed on day 2 and day 6 after infection ( $n=8$ per each group). (d) Lung histopathological analysis was performed on day 2 and day 7 after infection. H\&E-stained lung tissue at magnification of $\times 200$ (upper) and $\times 400$ (lower). Arrow showed neutrophils around bronchus. (e) The lung injury scores of lung sections from infectedWT and IL-36R ${ }^{-1-}$ mice at day 7 were evaluated as previously described ( $n=6$ per each group). Lung injury scoring system parameters include the presence of neutrophils in the alveolar space (i), neutrophils in the interstitial space (ii), hyaline membranes (iii), proteinaceous debris filling the airspaces (iv), and alveolar septal thickening (v). At least 20 random regions were scored $0-2$ independently and the final lung injury score was calculated as below; score $=((20 \times \mathrm{i})+(14 \times \mathrm{ii})+(7 \times \mathrm{iii})+(7 \times \mathrm{iv})+(2 \times \mathrm{v})) /($ number of fields $\times 100)$. (f) Lung permeability was quantified by albumin concentration in BAL fluid from 2 and 6 days after infection $\left(n=4\right.$ per each group). Data (means \pm s.d.) are representative of two independent experiment. ${ }^{\star} P<0.05$, ' $P<0.0001$, NS, not significant, compared with infected in WT mice or as indicated. BAL, bronchoalveolar lavage; IL-36, interleukin-36; H\&E, hematoxylin and eosin; WT, wild type.

day 6, albumin levels in BAL fluid were significantly reduced in IL-36R $\mathrm{R}^{-1-}$ mice compared with WT mice. Reduced alveolar permeability in influenza virus-infected IL-36 $\mathrm{R}^{-1-}$ mice was associated with a significant decrease in TUNEL positive apoptotic cells in IL-36 $\mathrm{R}^{-1-}$ mice compared with WT mice (see Supplementary Figure S2A,B). These data suggested that IL-36R ligands contributed to pathogenesis of lung injury and mortality induced by influenza virus despite a beneficial role in viral clearance.

\section{Reduced lung leukocyte influx and activation in IL-36R ${ }^{-I}$ mice during influenza virus infection}

We next quantified differences in lung inflammatory cell accumulation in WT and IL-36 $\mathrm{R}^{-1-}$ mice during influenza virus infection. No difference in number of total BAL leukocyte and proportion of monocytes/macrophages were observed in uninfected $\mathrm{WT}$ and IL-36 $\mathrm{R}^{-1-}$ mice at baseline. After inoculation of influenza virus, total number of lung leukocytes in BAL fluid increased in a time-dependent manner (Figure 5a). At day 2 and day 6, the number of total leukocyte, neutrophils, and monocytes/macrophages in BAL fluid was significantly reduced in $\mathrm{IL}^{-} 36 \mathrm{R}^{-/-}$mice as compared with their WT counterparts.
Lymphocytes, especially CD8 T cells, contribute to influenza virus clearance during infection. Murine lymphocytes including CD4 T cells, CD8 T cells, NK cells, and $\gamma \delta \mathrm{T}$ cells express the IL-36 receptor, and IL-36 cytokines can directly influence activation and proliferation of selected T-cell populations. ${ }^{25}$ We therefore examined the accumulation and activation of specific lymphocyte populations by flow cytometry. At day 2 of influenza virus infection, the numbers of $\mathrm{CD} 4+\mathrm{T}$ cells, CD8 $+\mathrm{T}$ cells, NK cells, NKT cells, and $\gamma \delta \mathrm{T}$ cells were significantly reduced in infected IL-36R $\mathrm{R}^{-1-}$ mice than in WT animals. Moreover, decreased numbers of activated lymphocyte populations, as identified by co-expression of activation marker CD69, were observed in IL-36R deficient mice. In contrast, total numbers and numbers of activated CD4 + and $\mathrm{CD} 8+\mathrm{T}$ cell were considerably greater in IL-36 $\mathrm{R}^{-1-}$ mice than in WT mice at day 6 (Figure $\mathbf{5 b}$ ).

\section{IL-36R ${ }^{-I-}$ mice exhibit reduced early cytokine and chemokine production during influenza virus infection}

Cytokines and chemokines contribute to control of viral replication via activation of both myeloid and parenchymal cells. However, these cytokines can also contribute to pathophysiology in patients with severe influenza virus infection. ${ }^{26}$ Therefore, we examined whether IL-36R genetic 

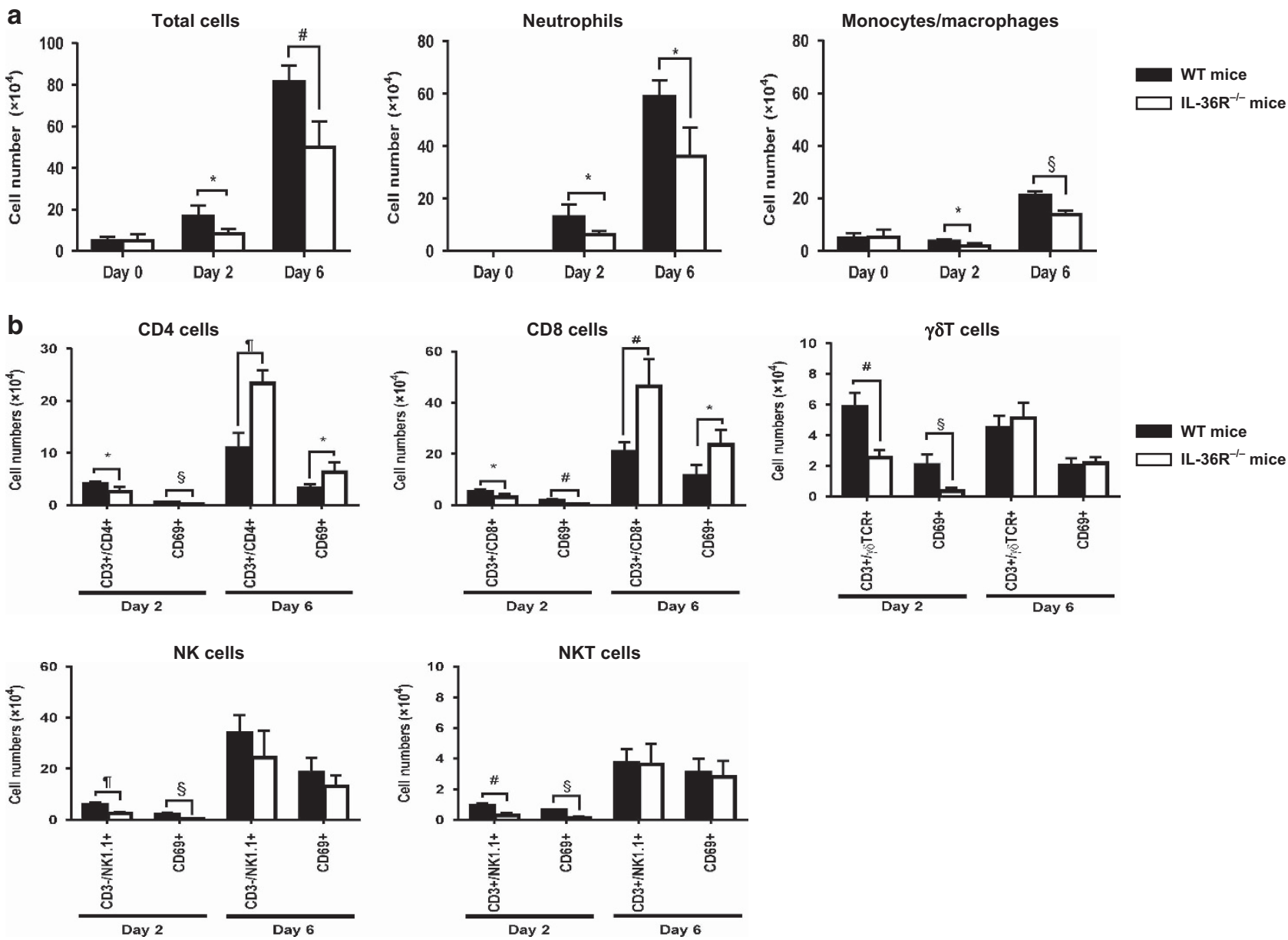

Figure 5 Effect of IL-36R gene deletion on lung leukocytes influx and lymphocyte activation during influenza virus infection. (a) BAL sample were collected at day 0 , day 2, and day 6 after influenza virus infection and the number of total cell, neutrophils, and monocytes and macrophages were counted. (b) The number of CD4 + T cells, CD8 + T cells, NK cells, NKT cells, and $\gamma \delta$ T cells, and CD69-positive lymphocytes was determined by flow cytometry. Lung digests were collected at day 2 and day 6 post-infection. Each group consisted of four mice. Data (means \pm s.d.) are representative of two independent experiments. ${ }^{*} P<0.05,{ }^{\#} P<0.01,{ }^{\S} P<0.001,{ }^{\circledR} P<0.0001$ as indicated. BAL, bronchoalveolar lavage; IL-36, interleukin-36.

deficiency altered cytokines/chemokine production during influenza virus infection. BAL samples were collected from influenza virus-infected mice at day 2 and day 6 . At day 2, BAL levels of IL-6, IL-17, KC/CXCL1, and IP-10/CXCL10 were significantly lower in IL-36 $\mathrm{R}^{-1-}$ mice compared with infected WT mice (Figure 6). By comparison, there was no difference in BAL fluid IFN- $\gamma$, TNF- $\alpha$, and CCR2/MCP-1 levels (see Supplementary Figure S2A) between WT and IL-36R ${ }^{-1-}$ mice during the course of infection. No difference in the levels of cytokines/chemokines was noted at day 6 .

\section{IL-36 $\alpha$ enhanced cytokine/chemokine production from AECs and PMs}

To examine whether IL-36 $\alpha$ might promote the production of cytokines and chemokines by cells present within the alveolus in either an autocrine or paracrine fashion, primary AECs and PMs were isolated from WT and IL-36R ${ }^{-1-}$ mice, then treated with mrIL-36 $\alpha$ for $24 \mathrm{~h}$, and the production of IL- 6 and KC quantitated. Cells from IL-36 $\mathrm{R}^{-1-}$ mice were used as a control to exclude non-specific stimulation (e.g. LPS contamination).
Treatment with rIL-36 $\alpha$ induced the production of IL- 6 and CXCL1/KC from WT AECs and PMs. This effect was dependent upon IL-36R signaling, as no induction of IL-6 and $\mathrm{KC}$ was observed in AECs and PMs isolated from IL-36R ${ }^{-1-}$ mice (Figure 7a,b).

Finally, using an in vitro model of cell-to-cell networking, we examined whether MPs released from influenza-infected AECs might stimulate the production of IL- 6 and KC by PMs in an IL-36R-dependent fashion. AECs isolated from WT mice were treated with or without influenza virus for $48 \mathrm{~h}$, then MPs isolated from CM by ultracentrifugation. We next incubated primary PMs isolated from WT and IL-36R ${ }^{-1-}$ mice with AECs-derived MPs. Because influenza virus-mediated MPs contained live influenza virus (Figure 3d,e), we first examined the production of IL- 6 and KC in response to influenza virus between PMs isolated from WT and IL-36R ${ }^{-1-}$ mice, and no differences were observed (data not shown). The induction of IL-6 and KC from PMs was significantly greater when incubated with MPs recovered from influenza virus-treated AECs as compared with MPs isolated from uninfected AEC. 

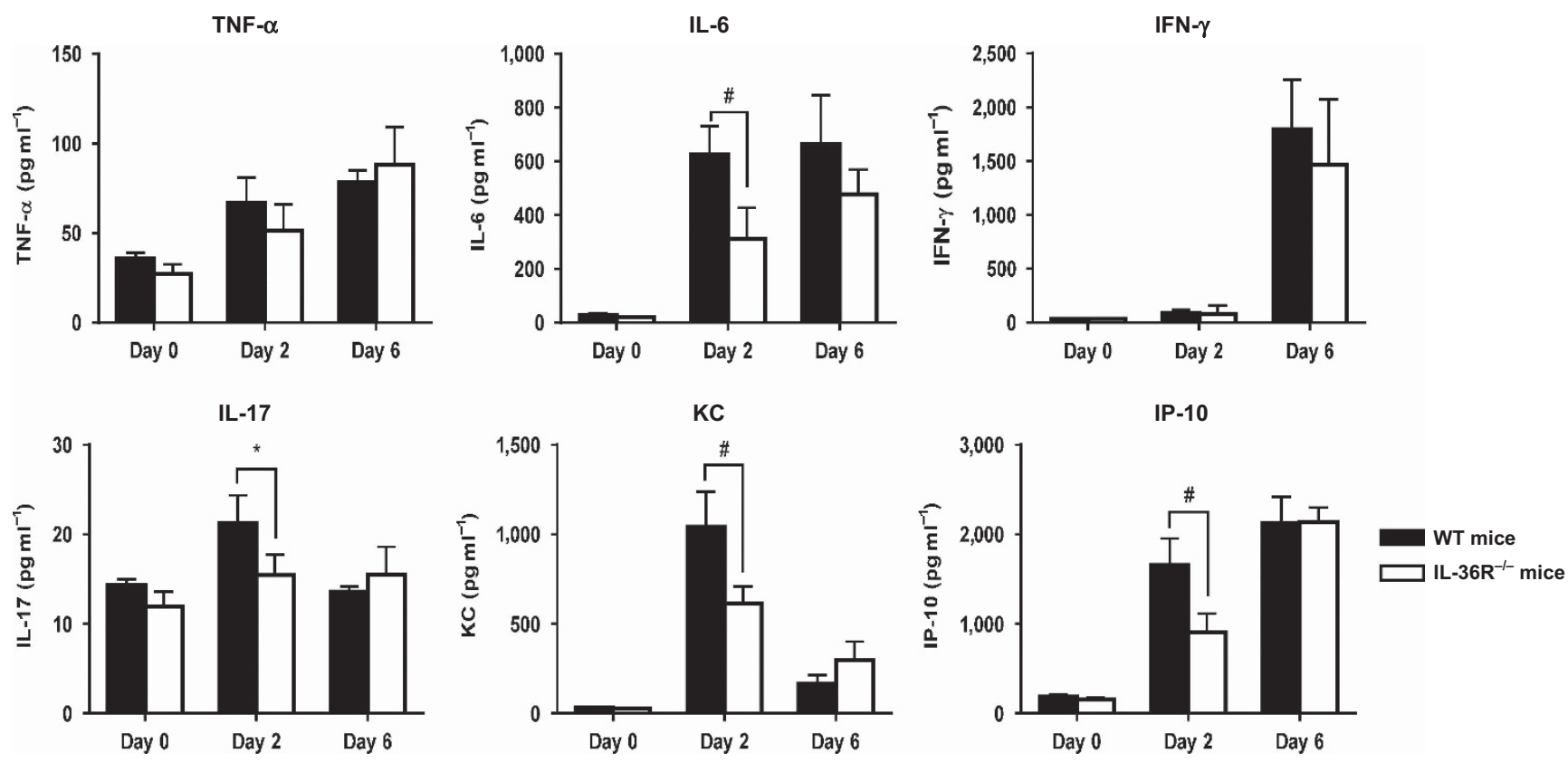

Figure 6 Effect of IL-36R gene deletion on the production of pro-inflammatory cytokines and chemokines during influenza virus infection. BAL sample were collected at day 0 , day 2 , and day 6 after influenza challenge. Levels of immunoreactive TNF- $\alpha$, IL-6, IFN- $\gamma$, IL-17, KC/CXCL1, and IP-10/CXCL10 were measured by ELISA. Each group is consisted of four mice. Data (means \pm s.d.) are representative of two independent experiments. ${ }^{*} P<0.05$, ${ }^{\#} P<0.01$ as indicated. BAL, bronchoalveolar lavage; ELISA, enzyme-linked immunosorbent assay; IL, interleukin; TNF- $\alpha$, tumor necrosis factor- $\alpha$.

Moreover, we found that the MP-stimulated production of IL-6 and $\mathrm{KC}$ from PMs was IL-36R dependent, as MPs failed to induce cytokine production in PMs isolated from IL-36R ${ }^{-1-}$ mice (Figure 7c).

\section{DISCUSSION}

Influenza virus-induced lung injury is characterized by the disruption of the alveolar-capillary membranes and pulmonary edema. ${ }^{26}$ Both bronchial and AECs play an important role in the immune response against influenza virus. If the host immune response and virus replication is not adequately controlled at infectious site, excessive inflammation can lead to epithelial cell death and tissue damage. In this study, we demonstrate that IL-36R ligands released from AECs and possibly other lung cells contribute to influenza virus-induced lung injury.

Influenza virus is single-stranded (ss) RNA virus, but produces double-stranded (ds) RNA intermediates during replication. TLR-3 and TLR-7 recognized ssRNA and dsRNA, respectively, and two distinct innate immune receptormediated signaling pathway, TLR-3-TRIF and TLR-7MyD88, induced production of type- 1 interferon or proinflammatory cytokines. ${ }^{27}$ We have previously demonstrated that the induction of IL-36 $\gamma$ in PMs by LPS and whole bacteria was MyD88 dependent. ${ }^{22}$ Our results indicate that TLR-4 and TLR-7, but not TLR-3, mediated the upregulation IL-36 $\alpha$ mRNA in AECs (Figure 2b), and suggest that MyD88-, rather than TRIF signaling is required for induction of IL-36 $\alpha$ message in AECs. It is known that secretion of IL- $1 \beta$ and IL-18 from influenza virus-infected cells depends on intracellular caspase- 1 activity via NLRP-3 inflammasome components. ${ }^{27}$ We observed that treatment with a caspase-1 inhibitor suppressed IL-36 $\alpha$ mRNA expression in response to influenza virus (Figure 2d), suggesting that induction of IL-36 $\alpha$ mRNA in influenza virus-infected AECs is regulated by caspase- 1 activity. Previous studies have demonstrated that caspase-3/7 activation induced by either ATP ${ }^{8,16}$ or TLR-3 $3^{17}$ signal was required for secretion of IL-36 cytokines. Our results are consistent with previous studies demonstrating the requirement for viral replication, indicating a contribution of both TLR-7 and TLR-3 signal for maximal secretion of IL-36 $\alpha$.

MPs have been detected in patients with sepsis and acute respiratory distress syndrome. These structures are released from both apoptotic and activated cells in response to various stimuli such as LPS, cytokines and cellular stress. During sepsis, MPs have been associated with endothelial injury and vascular tone through pro-inflammatory pathways, pro-coagulation activity, and both oxidative and nitrogen stress. ${ }^{21}$ However, little is known regarding the role of pathogen-mediated MPs in infectious disease. We found that influenza virus-induced IL$36 \alpha$ was contained within MPs shed from AECs (Figure 3b,c), as well as MPs in BAL fluid during influenza virus infection in vivo (Figure 1c). Furthermore, IL-36 $\alpha$ packaged within MPs enhanced the production of IL- 6 and KC by PMs raising the possibility of autocrine and paracrine networking between cells within the alveolar space (Figure 7c). In addition, we found that live influenza virus was packaged within virus-induced MPs (Figure 3c, d). Thus, MPs containing both IL-36 $\alpha$ and influenza virus together have the potential of amplifying 

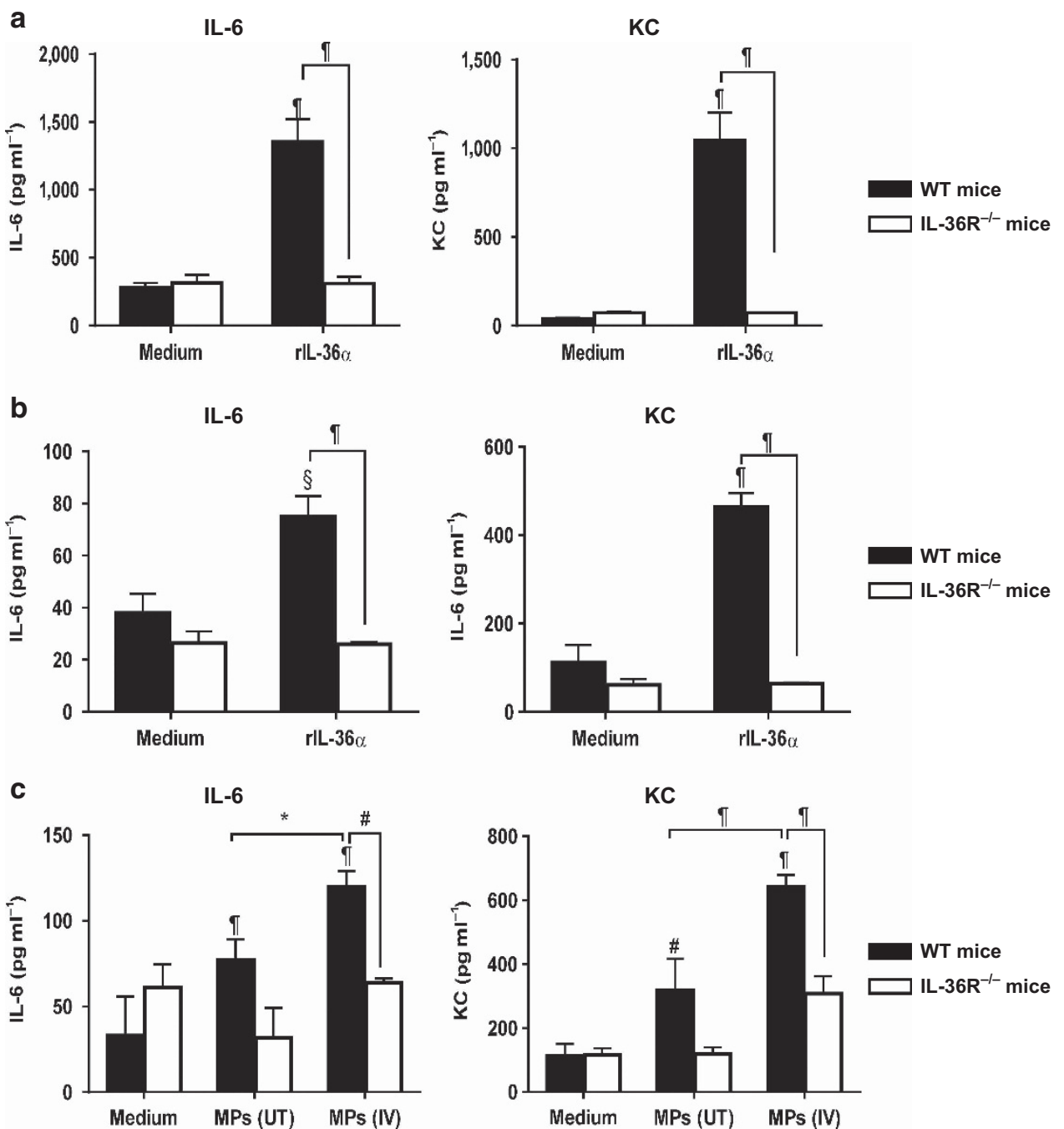

Figure 7 IL-36 $\alpha$ induced production of cytokine and chemokines from primary AECs and PMs. Primary AECs and PMs isolated from WT and IL-36R ${ }^{-/-}$ mice were treated with recombinant IL-36 $\alpha\left(100 \mathrm{ng} \mathrm{ml}^{-1}\right)$ for $24 \mathrm{~h}(\mathbf{a}, \mathbf{b})$. The production of IL-6 and KC by AECs (a) and PMs (b) were measured by ELISA. (c) AECs isolated from WT mice were treated with or without influenza virus. After $48 \mathrm{~h}$ incubation, MPs were isolated by ultracentrifugation method. Primary PMs isolated from WT and IL-36R ${ }^{-1-}$ mice were treated with influenza virus-mediated and untreated MPs for $24 \mathrm{~h}$. The production of IL-6 and KC in CM was measured by ELISA. rIL-36 $\alpha$, recombinant IL-36 $\alpha$; MPs (UT), MPs from untreated AECs; MP (IV), MPs from influenza virus-treated AECs. Data (means \pm s.d.). ${ }^{\star} P<0.05$, ${ }^{\#} P<0.01,{ }^{\S} P<0.001,{ }^{\circ} P<0.0001$ compared with medium or as indicated as indicated. AEC, alveolar epithelial cell; ELISA, enzyme-linked immunosorbent assay; IL-36 $\alpha$, interleukin36 $\alpha$; MP, microparticle; PM, PM, pulmonary macrophage: WT, wild type.

inflammatory responses in the lungs, which in the setting of influenza infection results in adverse consequences.

We found that lung injury was mitigated in IL-36R ${ }^{-1-}$ mice, as indicated by less albumin concentration (Figure $4 \mathbf{f}$ ), histological lung injury score (Figure 4d, e), and lung epithelial cell apoptosis (see Supplementary Figure 2A,B). Reduction in lung injury in IL-36R $\mathrm{R}^{-1-}$ mice was associated with significantly decreased early accumulation and activation of inflammatory cells, including monocytes, neutrophils, NK, and lymphocyte populations. Previous studies demonstrated that CCR2-positive exudative monocytes influx into alveolar space and contributed to lung epithelial cell damage through TRAILmediated apoptosis after influenza virus infection. ${ }^{28,29}$ However, we found no differences in the CCR2-ligand MCP-1 production or in the expression level of TRAIL mRNA in the lungs between influenza virus-infected lungs of WT and IL-36R $\mathrm{R}^{-1-}$ mice (see Supplementary Figure 3A,B). Macrophages and monocytes express IL-36 receptor, and changes in macrophage activational state may be IL-36dependent. However, there was no difference in NO activity, which is an activate marker of macrophages/ monocytes, between two groups (see Supplementary data S3C). These data suggest that the mechanism of reduced injury/ apoptosis of lung epithelial cells in influenza virus-infected IL-36R $\mathrm{R}^{-1-}$ mice is not due to differences in TRAIL-mediated apoptosis.

A reduction in neutrophil influx was noted in the lungs of IL-36R deficient mice during influenza infection (Figure 5a). Exuberant recruitment and activation of neutrophils is considered to play a key role in progression of acute lung 
injury/acute respiratory distress syndrome in several disease conditions. ${ }^{30}$ In influenza infection, neutrophils have been shown to promote epithelial-endothelial barrier damage by the production of neutrophils extracellular traps, ${ }^{31}$ reactive oxygen species, ${ }^{32}$ and myeloperoxidase. ${ }^{33} \mathrm{In}$ flu-induced acute respiratory distress syndrome patients, IL-17, CXCL8/ IL-8, and CXCL10/IP-10 have been shown to be elevated in serum and BAL fluid and levels correlated with disease severity. ${ }^{3,34} \mathrm{IL}-17$ and CXC chemokines are known to be key mediators of neutrophil migration. In addition, neutrophils express CXCR3, and IP-10/CXCL10 can stimulate the respiratory burst in neutrophils. ${ }^{35}$ IL-17 is known to be responsible for upregulation of CXCR2 ligands such as $\mathrm{KC}^{36}$ as well as the CXCR3 ligand IP- $10 .{ }^{37}$ In murine influenza infection, both IL-17 receptor and IL-17A-deficient mice showed increased survival rate and decreased lung injury, which occurred in association with reduced neutrophil accumulation. ${ }^{38,39}$ In addition, mice treated with IP-10 antibody and IP-10 KO mice attenuated the mortality of influenza virus induced lung injury. ${ }^{35,40}$ We found that the early production of IL-17, KC, and IP-10 at day 2 was significantly lower in IL-36 $\mathrm{R}^{-1-}$ mice as compared with influenza-infected WT mice. In influenza virus infection, the cellular source of IL-17 in the lungs is believed to be $\gamma \delta \mathrm{T}$ cells. ${ }^{38,41}$ These cells also express the IL-36 receptor, and stimulation with IL-36 cytokine directly promotes IFN- $\gamma$ production. ${ }^{25}$ Importantly, we found that the absolute number of $\gamma \delta \mathrm{T}$ cells and CD69 $+\gamma \delta \mathrm{T}$ cells in the lungs are much lower in IL-36R $\mathrm{R}^{-1-}$ compared with WT at day 2 post infection, which may account for attenuated production of IL-17 in IL-36 $\mathrm{R}^{-1-}$ mice after influenza virus infection. We also observed that IL-36 $\alpha$ directly induces KC production from AECs and PMs (Figure 7a,b). Since murine neutrophils do not express the IL-36 receptor, ${ }^{42}$ IL-36R ligands are unlikely to directly mediate neutrophil accumulation and activation.

Of interest, we observed impaired viral clearance in IL-36R $\mathrm{R}^{-1-}$ mice. Our results are in line with the findings of others showing that influenza virus induced mortality does not necessarily correlate with lung viral clearance. ${ }^{43-45} \mathrm{~A}$ fine balance exists between optimal immune clearance of virus and immune-mediated lung tissue damage. Neutrophils and exudate macrophages contribute to viral clearance, and reductions in these cell populations in IL-36R $\mathrm{R}^{-1-}$ mice may account for both attenuated lung injury responses and reduced viral clearance. A previous in vitro study demonstrated that IL-36 $\gamma$ directly promotes naïve $\mathrm{CD} 8+\mathrm{T}$ cells proliferation and differentiation to effector CD8 $+\mathrm{T}$ cells, ${ }^{25}$ thus IL-36 receptor ligands may be considered to contribute to virus clearance due to lymphocytes accumulation and antigen-specific activation at the infectious site. It is tempting to postulate that viral clearance was delayed in IL-36 $\mathrm{R}^{-1-}$ mice due to early reduction in the number of activated CD4 + and CD8 + T lymphocytes, cells known to contribute to viral clearance. On the other hand, at day 6 after infection, the number of total and CD69+ CD4 + and CD $8+\mathrm{T}$ cells was much greater in IL-36R ${ }^{-1-}$ mice. This paradoxical increase in CD4 and CD8 accumulation in IL-36Rdeficient mice at later time points remains unexplained. One possibility is that higher viral titers as a consequence of reduced early inflammatory cell accumulation (PMN, mononuclear cells) may drive lymphocyte accumulation/activation at later time points, which occurs in an IL-36-independent fashion. Finally, we observed reductions in number and activation of $\gamma \delta$ $\mathrm{T}$ cells and more modest reductions in NK and NKT cells at early time points in IL-36R-deficient mice, although the contribution of these cell populations to viral clearance and influenza-induced lung injury is less well defined. Regardless, our findings indicate that although IL-36 agonists promote antiviral clearance responses in the lung, they also promote deleterious lung injury culminating in death.

This study has some limitations. Most notably, we did not precisely identify the IL-36 receptor ligand responsible for the effects observed in our model. Because influenza infection did not stimulate IL-36 $\gamma$ secretion by virus-infected AECs and PMs, and mortality in IL-36 $\gamma$ KO mice did not differ from WT mice during influenza virus infection, we speculate that IL-36 $\alpha$ rather than IL-36 $\gamma$ plays a central role in influenza virusinduced lung injury. Unfortunately, the polyclonal IL-36 $\alpha \mathrm{Ab}$ generated for antigen detection was non-neutralizing and no IL-36 $\alpha \mathrm{KO}$ mice is yet available. In addition, there is some uncertainty as to cellular sources of IL-36 $\alpha$ other than AECs in our model. A recent study demonstrated that neutrophils expressed IL-36 cytokines in a murine autoimmune encephalitis model. ${ }^{42}$

In conclusion, our findings indicate that IL-36 agonists released from AECs and almost certainly other lung cells play a pivotal role in influenza virus-induced lung injury. Further studies will focus on identifying IL-36R ligands in humans with influenza virus-induced lung injury and correlation with relevant clinical outcomes.

\section{METHODS}

Mice. Specific pathogen-free age- and sex-matched C57BL/6 mice were purchased from The Jackson Laboratory (Bar Harbor, ME). IL$36 \mathrm{R}^{-/-}$mice on C57BL/6 background were provided by Jennifer Towne from Amgen (Thousand Oaks, CA). ${ }^{10}$ A colony of IL-36 $\gamma^{-1-}$ mice bred on a C57BL/6 background was established at the University of Michigan (Ann Arbor, MI). ${ }^{22}$ All mice were housed in specific pathogen-free conditions within the University of Michigan Animal Care Facility. Animal studies were reviewed and approved by the University Committee on Use and Care of Animals at the University of Michigan.

Virus infection in vivo. Influenza A/Puerto Rico/8/34 virus was used for all of experiments. For intranasal infection, mice were anesthetized by intraperitoneal injection of ketamine and xylazine and then infected by intranasal administration of $2 \times 50 \%$ lethal dose $\left(\mathrm{LD}_{50}\right.$ : $\left.40 \mathrm{pfu}\right)$ of virus in $30 \mu \mathrm{l}$ of saline.

Bronchoalveolar lavage and lung sampling. Mice were killed by $\mathrm{CO}_{2}$ inhalation. The trachea was exposed and cannulated with a $22 \mathrm{G}$ intravenous catheter. BAL was performed with $1.5 \mathrm{ml}$ phosphatebuffered saline (PBS) (three aliquot $500 \mu \mathrm{l}$ of PBS), and then pulmonary circulation was rinsed by $1 \mathrm{ml}$ PBS. Right lungs were harvested to determine the virus titer and stored at $-80^{\circ} \mathrm{C}$. Left lungs were harvested for RNA extraction, immediately snap-frozen in liquid nitrogen. After a collection of leukocytes in BAL fluids, cytospin (1000 r.p.m. $\times 5 \mathrm{~min}$ ) preparations were made from each sample and stained with modified Wright stain. Differential cell counts of 
neutrophils and monocytes and macrophages were obtained for at least 400 cells counts in each sample at a magnification of $\times 1,000$.

Histopathological examination. Lungs and trachea were removed from killed animals and inflated at $20 \mathrm{~cm} \mathrm{H}_{2} \mathrm{O}$ with $4 \%$ paraformaldehyde through trachea, and fixed for paraffin embedding. All lungs were sectioned and stained with hematoxylin and eosin. Quantitative analysis of tissue injury was measured using the lung injury scoring system as described. ${ }^{24}$ Lung injury scoring system parameters include neutrophils in the alveolar space (A), neutrophils in the interstitial space (B), hyaline membranes $(C)$, proteinaceous debris filling the airspaces (D), and alveolar septal thickening (E). At least 20 random regions were scored $0-2$ independently at a magnification of $\times 400$ in a blinded fashion. The final lung injury score per each lungs was calculated as below: score $=((20 \times \mathrm{A})+(14 \times$ B) $+(7 \times \mathrm{C})+(7 \times \mathrm{D})+(2 \times \mathrm{E})) /($ number of fields $\times 100)$.

Virus titer. Madin-Darby canine kidney (MDCK) cells were plated at $1 \times 10^{6}$ cells in a flat bottomed six-well plate $24 \mathrm{~h}$ before infection. Supernatant from right lung homogenates serially diluted were used to infect the MDCK cells at $37^{\circ} \mathrm{C}$ for $1 \mathrm{~h}$. The cells were subsequently overlaid with Dulbecco's Modified Eagle Medium mixed 0.4\% agarose (Sigma-Aldrich, St Louis, MO) in the presence of $0.5 \mu \mathrm{g} \mathrm{ml}^{-1}$ Trypsin (Sigma-Aldrich) and incubated for $96 \mathrm{~h}$. The plaques were visualized by staining the cells with crystal violet.

Total lung leukocyte. Lung leukocytes were prepared as described in our previous study. ${ }^{46}$ Briefly, lungs were homogenated in $15 \mathrm{ml}$ of digestion buffer (RPMI1640 medium supplemented with 5\% fetal calf serum and containing $1 \mathrm{mg} \mathrm{m}^{-1}$ collagenase (Roche Diagnostics, Mannheim, Germany) and $30 \mu \mathrm{g} \mathrm{ml}^{-1}$ DNase I (Sigma-Aldrich)) per lung and enzymatically digested for $60 \mathrm{~min}$ at $37^{\circ} \mathrm{C}$. Tissue fragments were removed by passing the mesh. Total lung cell pellet was resuspended in $4 \mathrm{ml}$ of $40 \%$ (v/v) Percoll (Pharmacia, Uppsala, Sweden) and layered onto $4 \mathrm{ml}$ of $80 \%(\mathrm{v} / \mathrm{v})$ Percoll. After centrifugation at 2,000 r.p.m. for $20 \mathrm{~min}$ at room temperature, the cells were collected.

Murine PM and AEC isolation and culture. Murine PMs and type II AECs were isolated using the method described previously. ${ }^{47}$ Briefly, PMs were isolated from dispersed lung homogenates by adherence purification and plated at a density $5 \times 10^{5}$ cells per $\mathrm{ml}^{-1}$. For the isolation of murine AECs, the pulmonary vasculature was perfused. The lungs were filled via the trachea with $1.5 \mathrm{ml}$ dispase (Worthington, Lakewood, NJ), then $1.5 \mathrm{ml}$ of low-melting point agarose and finally placed in ice cold PBS. The lungs were submerged in dispase for $45 \mathrm{~min}$ at $24^{\circ} \mathrm{C}$ before the lung tissue was teased from the airways and minced in Dulbecco's Modified Eagle Medium with $0.01 \%$ DNase. After swirling for $15 \mathrm{~min}$, followed by passage through a series of nylon filters, the cell suspension was collected by centrifugation and incubated with biotinylated Abs (anti-CD32 and anti-CD45; BD Pharmingen, San Diego, CA). After incubation with streptavidincoated magnetic particles, myeloid cells were removed using a magnetic tube separator. Mesenchymal cells were removed by overnight adherence in a Petri dish and the resulting non-adherent cells were plated on plastic dishes coated with fibronectin. Previous work has shown that the day 3 time point has $>90 \%$ pure AECs. ${ }^{47}$ These cells were treated with influenza virus at a multiplicity of infection of $10-0.1$, LPS $\left(1 \mu \mathrm{g} \mathrm{ml}^{-1}\right)$ (Sigma-Aldrich), TLR-3 ligand: poly(I:C) $\left(25 \mu \mathrm{g} \mathrm{ml}^{-1}\right)$, TLR-7 ligand: R843 $\left(25 \mu \mathrm{g} \mathrm{ml}^{-1}\right)$ (Invivogen, San Diego, CA) and recombinant IL-36 $\alpha$ (rIL-36 $\alpha$; R\&D Systems Minneapolis, MN) (100 $\left.\mathrm{n} \mathrm{ml}^{-1}\right)$. For blockade of caspase-1 and caspase-3/7, inhibitor of Caspase-1 and Caspase-3/7 (Cayman Chemical, Ann Arbor, MI), which were soluble in dimethyl sulfoxide (add vehicle control), was added to cultures $1 \mathrm{~h}$ before stimulation with medium and influenza virus. Final concentrations were $20 \mu \mathrm{M}$ (caspase-1 inhibitor) and $100 \mu \mathrm{M}$ (caspase-3/7 inhibitor).

Isolation of microparticles and EXs in vivo and in vitro. We used a standard three-step differential centrifugation method to isolate MPs and EXs in vivo and in vitro samples, as previously described. ${ }^{22} \mathrm{BAL}$ fluid and CM was centrifuged to remove cells and large debris at 3,000 r.p.m. for $10 \mathrm{~min}$ and stored these samples at $-80^{\circ} \mathrm{C}$ overnight. Each sample supernatant was centrifuged at 17,000 r.p.m. for $45 \mathrm{~min}$ to obtain the MPs. After collecting the supernatant, the pellet containing the MPs fraction was suspended in $100 \mu \mathrm{l}$ of lysis buffer or PBS. For the final step, each supernatant was centrifuged at 28,000 r.p.m. for $90 \mathrm{~min}$ to isolate EXs. The pellet was suspended in $100 \mu$ l of lysis buffer or PBS. In some experiments, MPs were washed twice by Dulbecco's Modified Eagle Medium and suspended $100 \mu \mathrm{l}$ of Dulbecco's Modified Eagle Medium and cultured with MDCK cells. MPs, EXs, and the post-spin fluid in which MPs and EXs were removed by ultracentrifugation were stored in $-80^{\circ} \mathrm{C}$.

Flow cytometry analysis. Cells, isolated from whole lung or MPs and EXs as described above, were stained with the following FITC- or PE- or PerCP-Cy5.5 labeling antibodies to CD3, CD4, CD8, NK1.1, $\gamma \delta$ TCR, CD69, and Annexin V (BD Pharmigen, San Jose, CA) and analyzed by Attune Acoustic Focusing Cytometer (Thermo Scientific-Applied Biosystems, Foster City, CA). Isotype control was used for all the samples. MPs and EXs were defined as Annexin V-positive cells. To measure the size of MPs and EXs, we used calibration beads from 0.1 to $3 \mu \mathrm{m}$ in diameter, as previously described. $^{48,49}$

Determination of cytokine production by enzyme-linked immunosorbent assay. IL-36 $\alpha$, secreted in fluid or contained in MPs and EXs, was measured by sandwich ELISA protocol. Other cytokines/ chemokines (TNF- $\alpha$, IL-6, IFN- $\gamma$, IL-17, KC and IP-10; R\&D systems) and albumin (Albumin Quantification Kit: Bethyl Laboratories, Montgomery, TX) were quantified using a modified double-ligand method as described.

RNA isolation and real-time PCR. RNA was isolated and real-time quantitative RT-PCR was performed by AB Step One plus Real-Time PCR System (Thermo Scientific-Applied Biosystems). Predesigned primer and probes of targeted molecules and $\beta$-actin as a control were purchased from Integrated DNA Technologies (Coralville, IA). Quantification of $\beta$-actin and target genes in each sample set was performed by the standard curve method.

Western blotting. Cells were digested by RIPA buffer (Sigma-Aldrich) plus protease inhibitors and gels were subjected to electrophoresis as previously described. ${ }^{47}$ Membranes were incubated with primary antiIL-36 $\alpha$ antibody (R\&D systems; diluted 1:250), anti-Influenza virus M2 antigen antibody (Santa Cruz Biotechnology, Santa Cruz, CA; diluted 1: 1,000), or $\beta$-actin (Sigma-Aldrich; diluted 1:5,000), blots were incubated with a secondary antibody linked to HRP, and the signals were developed with an ECL (SuperSignal West Pico Substrate; Pierce Biotechnology, Rockford, IL).

Statistical analysis. Descriptive statics, such as means and standard deviations, were collected. The difference in survival rates was evaluated by the log rank test (Mantel-Cox). Two sets of values were evaluated by the Student's $t$-test, and more than three sets of value were evaluated by ANOVA, followed by the Turkey's multiple comparison test. Data analysis was conducted using GraphPad prism 6 (GraphPad Software, La Jolla, CA). A $P$-value of $<0.05$ was considered satirically significance.

SUPPLEMENTARY MATERIAL is linked to the online version of the paper at http://www.nature.com/mi

\section{ACKNOWLEDGMENTS}

This work was supported by Tohoku University Leading Young Researcher Overseas Visit Program (to T.A.), Japanese Society for the Promotion of Science Grant-in-Aid for Scientific Research 25860825 (to T.A.), and National Institutes of Health/National Heart Lung, and Blood Institute Grants NIH/NHLBI R01 HL123515 and R01 HL097564 (to T.J.S.). 


\section{DISCLOSURE}

The authors declared no conflict of interest.

(c) 2017 Society for Mucosal Immunology

\section{REFERENCES}

1. Dawood, F.S. et al. Estimated global mortality associated with the first 12 months of 2009 pandemic influenza A H1N1 virus circulation: a modelling study. Lancet Infect. Dis. 12, 687-695 (2012).

2. de Jong, M.D. et al. Fatal outcome of human influenza A (H5N1) is associated with high viral load and hypercytokinemia. Nat. Med. 12, 12031207 (2006).

3. Chi, Y. et al. Cytokine and chemokine levels in patients infected with the novel avian influenza A (H7N9) virus in China. J. Infect. Dis. 208, 1962-1967 (2013).

4. To, K.K. et al. Delayed clearance of viral load and marked cytokine activation in severe cases of pandemic H1N1 2009 influenza virus infection. Clin. Infect. Dis. 50, 850-859 (2010).

5. Gabay, C. \& Towne, J.E. Regulation and function of interleukin-36 cytokines in homeostasis and pathological conditions. J. Leuk. Biol. 97, 645-652 (2015).

6. Carrier, Y. et al. Inter-regulation of Th17 cytokines and the IL-36 cytokines in vitro and in vivo: implications in psoriasis pathogenesis. J. Invest. Dermatol. 131, 2428-2437 (2011).

7. Vigne, S. et al. IL-36 signaling amplifies Th1 responses by enhancing proliferation and Th1 polarization of naive CD4 + Tcells. Blood 120, 34783487 (2012).

8. Johnston, A. et al. IL-1F5, -F6, -F8, and -F9: a novel IL-1 family signaling system that is active in psoriasis and promotes keratinocyte antimicrobial peptide expression. J. Immunol. 186, 26132622 (2011).

9. Marrakchi, S. et al. Interleukin-36-receptor antagonist deficiency and generalized pustular psoriasis. N. Engl.J. Med. 365, 620-628 (2011).

10. Blumberg, H. et al. Opposing activities of two novel members of the IL-1 ligand family regulate skin inflammation. J. Exp. Med. 204, 2603-2614 (2007).

11. Tortola, L. et al. Psoriasiform dermatitis is driven by IL-36-mediated DCkeratinocyte crosstalk. J. Clin. Invest. 122, 3965-3976 (2012).

12. Chustz, R.T. et al. Regulation and function of the IL-1 family cytokine IL-1F9 in human bronchial epithelial cells. Am. J. Respir. Cell Mol. Biol. 45, 145-153 (2011).

13. Vos, J.B. et al. Transcriptional response of bronchial epithelial cells to Pseudomonas aeruginosa: identification of early mediators of host defense. Physiol. Genomics 21, 324-336 (2005).

14. Ramadas, R.A., Ewart, S.L., Iwakura, Y., Medoff, B.D. \& LeVine, A.M. IL-36alpha exerts pro-inflammatory effects in the lungs of mice. PLoS One 7, e45784 (2012).

15. Ramadas, R.A., Ewart, S.L., Medoff, B.D. \& LeVine, A.M. Interleukin-1 family member 9 stimulates chemokine production and neutrophil influx in mouse lungs. Am. J. Respir. Cell Mol. Biol. 44, 134-145 (2011).

16. Martin, U. et al. Externalization of the leaderless cytokine IL-1F6 occurs in response to lipopolysaccharide/ATP activation of transduced bone marrow macrophages. J. Immunol. 183, 4021-4030 (2009).

17. Lian, L.H., Milora, K.A., Manupipatpong, K.K. \& Jensen, L.E. The doublestranded RNA analogue polyinosinic-polycytidylic acid induces keratinocyte pyroptosis and release of IL-36gamma. J. Invest. Dermatol. 132, 1346-1353 (2012).

18. Gombault, A., Baron, L. \& Couillin, I. ATP release and purinergic signaling in NLRP3 inflammasome activation. Front. Immunol. 3, 414 (2012).

19. Eltom, S. et al. Respiratory infections cause the release of extracellular vesicles: implications in exacerbation of asthma/COPD. PLoS One 9, e101087 (2014).

20. Sakaki, H. et al. P2X4 receptor regulates $P 2 X 7$ receptor-dependent $\mathrm{IL}-1$ beta and IL-18 release in mouse bone marrow-derived dendritic cells. Biochem. Biophys. Res. Commun. 432, 406-411 (2013).
21. Reid, V.L. \& Webster, N.R. Role of microparticles in sepsis. Br. J. Anaesth. 109, 503-513 (2012).

22. Kovach, M.A. et al. IL-36gamma is secreted in microparticles and exosomes by lung macrophages in response to bacteria and bacterial components. J. Leuk. Biol. 100, 413-421 (2016).

23. Takeuchi, O. \& Akira, S. Pattern recognition receptors and inflammation. Cell 140, 805-820 (2010).

24. Matute-Bello, G. et al. An official American Thoracic Society workshop report: features and measurements of experimental acute lung injury in animals. Am. J. Respir. Cell Mol. Biol. 44, 725-738 (2011).

25. Wang, X. et al. IL-36gamma transforms the tumor microenvironment and promotes type 1 lymphocyte-mediated antitumor immune responses. Cancer Cell 28, 296-306 (2015).

26. Short, K.R., Kroeze, E.J., Fouchier, R.A. \& Kuiken, T. Pathogenesis of influenza-induced acute respiratory distress syndrome. Lancet Infect. Dis. 14, 57-69 (2014).

27. Iwasaki, A. \& Pillai, P.S. Innate immunity to influenza virus infection. Nat. Rev. Immunol. 14, 315-328 (2014).

28. Herold, S. et al. Lung epithelial apoptosis in influenza virus pneumonia: the role of macrophage-expressed TNF-related apoptosis-inducing ligand. J. Exp. Med. 205, 3065-3077 (2008).

29. Hogner, K. etal. Macrophage-expressed IFN-beta contributes to apoptotic alveolar epithelial cell injury in severe influenza virus pneumonia. PLoS Pathog. 9, e1003188 (2013).

30. Grommes, J. \& Soehnlein, O. Contribution of neutrophils to acute lung injury. Mol. Med. 17, 293-307 (2011).

31. Narasaraju, T. et al. Excessive neutrophils and neutrophil extracellular traps contribute to acute lung injury of influenza pneumonitis. Am. J. Pathol. 179, 199-210 (2011).

32. Snelgrove, R.J., Edwards, L., Rae, A.J. \& Hussell, T. An absence of reactive oxygen species improves the resolution of lung influenza infection. Eur. J. Immunol. 36, 1364-1373 (2006).

33. Sugamata, R. et al. Contribution of neutrophil-derived myeloperoxidase in the early phase of fulminant acute respiratory distress syndrome induced by influenza virus infection. Microbiol. Immunol. 56, 171-182 (2012).

34. Hagau, N. et al. Clinical aspects and cytokine response in severe H1N1 influenza A virus infection. Crit. Care 14, R203 (2010).

35. Ichikawa, A. et al. CXCL10-CXCR3 enhances the development of neutrophil-mediated fulminant lung injury of viral and nonviral origin. Am. J. Respir. Crit. Care Med. 187, 65-77 (2013).

36. Hartupee, J., Liu, C., Novotny, M., Li, X. \& Hamilton, T. IL-17 enhances chemokine gene expression through mRNA stabilization. J. Immunol. 179, 4135-4141 (2007).

37. Khader, S.A. et al. IL-23 and IL-17 in the establishment of protective pulmonary $\mathrm{CD} 4+\mathrm{T}$ cell responses after vaccination and during Mycobacterium tuberculosis challenge. Nat. Immunol. 8, 369-377 (2007).

38. Crowe, C.R. et al. Critical role of IL-17RA in immunopathology of influenza infection. J. Immunol. 183, 5301-5310 (2009).

39. Li, C. et al. IL-17 response mediates acute lung injury induced by the 2009 pandemic influenza A (H1N1) virus. Cell Res. 22, 528-538 (2012).

40. Wang, W. et al. Monoclonal antibody against CXCL-10/IP-10 ameliorates influenza A (H1N1) virus induced acute lung injury. Cell Res. 23, 577-580 (2013).

41. Hamada, H. et al. Tc17, a unique subset of CD8 T cells that can protect against lethal influenza challenge. J. Immunol. 182, 3469-3481 (2009).

42. Bozoyan, L., Dumas, A., Patenaude, A. \& Vallieres, L. Interleukin36 gamma is expressed by neutrophils and can activate microglia, but has no role in experimental autoimmune encephalomyelitis. J. Neuroinflammation 12, 173 (2015).

43. Cook, D.N. et al. Requirement of MIP-1 alpha for an inflammatory response to viral infection. Science 269, 1583-1585 (1995).

44. Dawson, T.C., Beck, M.A., Kuziel, W.A., Henderson, F. \& Maeda, N. Contrasting effects of CCR5 and CCR2 deficiency in the pulmonary inflammatory response to influenza A virus. Am. J. Pathol. 156, 1951-1959 (2000).

45. Carey, M.A. et al. Contrasting effects of cyclooxygenase-1 (COX-1) and COX-2 deficiency on the host response to influenza A viral infection. J. Immunol. 175, 6878-6884 (2005). 
46. Aoyagi, T. et al. Activation of pulmonary invariant NKT cells leads to exacerbation of acute lung injury caused by LPS through local production of IFN-gamma and TNF-alpha by Gr-1 + monocytes. Int. Immunol. 23, 97-108 (2011).

47. Seki, M. et al. Critical role of IL-1 receptor-associated kinase-M in regulating chemokine-dependent deleterious inflammation in murine influenza pneumonia. J. Immunol. 184, 1410-1418 (2010).
48. Densmore, J.C. et al. Endothelium-derived microparticles induce endothelial dysfunction and acute lung injury. Shock 26, 464-4712006).

49. Dinkla, S., Brock, R., Joosten, I. \& Bosman, G.J. Gateway to understanding microparticles: standardized isolation and identification of plasma membrane-derived vesicles. Nanomedicine $\mathbf{8}$, 1657-1668 (2013). 